\title{
Pulsating variable stars and large spectroscopic surveys
}

\author{
Peter De Cat ${ }^{1, \star}$ \\ ${ }^{1}$ Royal Observatory of Belgium, Ringlaan 3, 1180 Brussels, Belgium
}

\begin{abstract}
In the past decade, the research of pulsating variable stars has taken a giant leap forward thanks to the photometric measurements provided by space missions like Most, CoRoT, Kepler/K2, and BRITE. These missions have provided quasi uninterrupted photometric time-series with an ultra-high quality and a total length that is not achievable from Earth. However, many of the success stories could not have been told without ground-based spectroscopic follow-up observations. Indeed, spectroscopy has some important assets as it can provide (more) accurate information about stellar parameters (like the effective temperature, surface gravity, metallicity, and abundances that are mandatory parameters for an in-depth asteroseismic study), the radial velocity (that is important for the detection of binaries and for the confirmation of cluster membership, if applicable), and the projected rotational velocity (that allows the study of the effects of rotation on pulsations). Fortunately, several large spectroscopic surveys are (becoming) available that can be used for these purposes. For some of these surveys, sub-projects have been initiated with the specific goal to complement space-based photometry. In this review, several spectroscopic surveys are introduced and compared with each other. We show that a large amount of spectroscopic data is (becoming) available for a large variety of objects.
\end{abstract}

\section{Introduction}

How can large spectroscopic surveys help the research of pulsating variable stars? Or, in other words, are they useful for the gathering of the required input for in-depth asteroseismic studies? A first requirement for asteroseismology is the observation of a large number of pulsation frequencies. Each pulsation frequency reveals information of a specific internal layer. So the more pulsation frequencies can be observed, the more complete the fingerprint of the stellar interior will be. Therefore, there is a need for time-series of high-quality data (to detect low amplitude variations) with a sufficiently long time-base (to cover the beat periods) in photometry (brightness variations) and/or in spectroscopy (radial velocity and/or line-profile variations). Space-based observations from past and ongoing missions like Most ([1]), CoRoT ([2]), Kepler/K2 ([3, 4]), and BRITE ([5]) have already proven their usefulness and we still have some high hopes for (future) space-missions like GaIa ([6]), Tess ([7]), and Plato ([8]). GaIA is the only mission from this list that also provides spectroscopic observations.

The second requirement is that the observed pulsation modes need to be identified. It means that their degree $\ell$ and azimuthal number $m$ should be known. In the most fortunate cases, quasi-regular spacings in either frequency (for pressure modes; [9]) or in period (for gravity modes; [10]) can be detected, making it possible to read the value of $\ell$ from the ridges in the corresponding Échelle

\footnotetext{
^Peter.DeCat@oma.be
} 
diagram (e.g., [10]). However, for most types of pulsating stars, the mode identification needs to be done by using techniques in multi-colour photometry (determination of $\ell$ only; [11]) and/or highresolution, high-quality spectroscopy (full identification; [12, 13]). Unfortunately, the data provided by large spectroscopic surveys are in general not appropriate for this purpose.

The third requirement is that accurate values for stellar parameters like the effective temperature (" $T_{\text {eff }}$ "), surface gravity (" $\log g$ "), metallicity (“ $[\mathrm{M} / \mathrm{H}]$ ]), projected rotational velocity (" $v \sin i$ "), and chemical abundances are needed to pinpoint the best model amongst those that are fitting the observed frequency spectrum. All these parameters can already be derived from one spectrum of sufficient resolution and quality. Large spectroscopic surveys can be extremely important in this respect. The resulting accurate position in the Hertzsprung-Russell (H-R) diagram can also be used for the confirmation of pulsation class membership or for the detection of new types of pulsating stars (like the mysterious star OGLE-GD-DSCT-0058; [14]). For some of the pulsation mechanisms, the driving of the modes heavily depends on the amount of metals that are present in the stellar interior. This is nicely illustrated by the B8.3 v star KIC10526294 for which only half of the observed gravity modes are driven in models with standard metal mixtures ([15]). Therefore, the importance of accurate values for especially the metallicity and chemical abundances must not be underestimated for making progress in the understanding of the physics of the stellar interior.

Finally, values of the radial velocity (" $v_{\mathrm{rad}}$ ") can give extra useful input for asterseismic modeling. A few $v_{\text {rad }}$ values could already be sufficient to detect multiple systems. Knowledge of their orbit can lead to constraints on the mass, the inclination and the distance of the components. A single $v_{\text {rad }}$ value could already be sufficient to confirm cluster membership. As cluster stars are formed from the same molecular cloud, they all have a common age, composition and distance that can be used in the asteroseismic investigation. In this respect, large spectroscopic surveys can be a goldmine too.

In what follows, a brief overview of large spectroscopic surveys is given. In this context, a spectroscopic survey is considered as large when at least 10000 objects are observed. Surveys only focusing on galaxies were omitted. We first present past and currently ongoing surveys (Sect. 2) before introducing a few surveys that are planned for the coming years (Sect. 3). We end with the summary and conclusions in Section 4.

\section{Past and ongoing large spectroscopic surveys}

\subsection{Xinglong Observatory (China)}

\section{LAMOST}

The Large Sky Area Multi-Object Fiber Spectroscopic Telescope (Lamost; Table 1; http://www . lamost.org; [16, 17]) is a new 4-m telescope located in the Xinglong Observatory (China) in the northern hemisphere. It is a unique instrument that combines a large aperture (4 meter) with a wide field-of-view ("FoV"; circular region with a diameter of 5 degrees on the sky) that is covered with 4000 optical fibers. It is the ideal tool to obtain spectra with a low spectral resolution $(R \sim 1800)$ in the optical $(370-900 \mathrm{~nm})$ for a large number of objects in a homogeneous and efficient way. The Lamost ExtraGAlactic Survey (Legas) and the Lamost Experiment for Galactic Understanding and Exploration (LEGUE; [18]) were the two initial scientific driving forces for the LAMOsT project. The survey aims to observe more than 5000000 objects (stars, galaxies and quasars) for which $r<17.8 \mathrm{mag}$ is a realistic magnitude limit. However, it was soon realized that the observation of the FoV of the nominal Kepler mission with the LAMosT would be a pure scientific goldmine, being a win-win opportunity for both communities: it would provide the Kepler community with the data needed for a homogeneous spectroscopic determination of stellar parameters for objects observed by Kepler while 
Table 1. Characteristics of the large spectroscopic survey conducted at the Xinglong Observatory (XING; China) in the northern hemisphere with the 4.0-m Large Sky Area Multi-Object Fiber Spectroscopic Telescope

(LAmost).

\begin{tabular}{|l|c|}
\hline Name & LAMOST \\
\hline Telescope & $4.0-\mathrm{m}$ XING \\
Fibers & $\# 4000$ \\
Wavelengths & $370-900 \mathrm{~nm}$ \\
Resolution & $\sim 1800$ (fixed) \\
Targets & $>5000000$ stars, galaxies \& quasars \\
& $(r<17.8 \mathrm{mag})$ \\
Date range & $2011-2017$ \\
\hline
\end{tabular}

the Lamost community could benefit from high-precision values for stellar parameters for Kepler objects derived from data obtained elsewhere to calibrate the LAmost results. Therefore, the proposal of the Lamost-Kepler project ([19]) was well received in 2010 and the first observations for the project were already done during the test phase of the LAmost in early 2011. The scientific observations of the pilot survey began on October 24, 2011 while the regular survey started about one year later, on September 28, 2012. So far, observations on 45 nights have led to 198727 Lamost spectra of stars within the Kepler FoV. The 101086 spectra obtained before October 2014 have been analyzed by three groups leading to atmospheric parameters and spectral classification for 51406 and 63149 stars, respectively ([20-22]). The accuracy of the derived $T_{\text {eff }}, \log g$, and $[\mathrm{Fe} / \mathrm{H}]$ values amounts to $\sim 3.5 \%$, $\sim 0.3$ dex and $\sim 0.2$ dex, respectively. Since the end of 2015, five K2 fields have been observed with the Lamost. Up to now, 96233 Lamost spectra have been collected and they were used to derive the stellar atmospheric parameters for 59213 stars ([23]). The first regular survey of the LAMost will end in June 2017. For the next phase, one of the options is to equip the Lamost with medium resolution spectrographs.

\subsection{Apache Point Observatory (New Mexico, USA)}

There are several large spectroscopic surveys for which the observations were/are being done from the Apache Point Observatory (New Mexico, USA; Table 2) in the northern hemisphere. They all use the 2.5-m Sloan Foundation Telescope having a circular FoV with a diameter of $3 \mathrm{deg}\left(\sim 7 \mathrm{deg}^{2}\right)$.

\section{SEGUE-1 $\mathcal{E}$ SEGUE-2}

The Sloan Extension for Galactic Understanding and Exploration (SEGUE; Table 2, top left; http: //www.sdss.org/surveys/segue/) was the first large spectroscopic survey that used this telescope. It was combined with a spectrograph with 640 fibers to obtain spectra with a resolution of $\sim 2000$ with a typical signal-to-noise ratio ("SNR") of 25 in the optical wavelength range $(385-920 \mathrm{~nm})$. The focus of the first phase (SEGUE-1; [24]) was on the exploration of the structure of the Milky Way by observing 240000 stars with a $g$-magnitude below 19 and covering a surface area of $1438 \mathrm{deg}^{2}$. These observations were done in dark and gray time from the winter of 2004 until the winter of 2008. During the second phase (SEGUE-2), the focus shifted to stars in the halo with distances from 10 to $60 \mathrm{kpc}$. A total of 118151 stars with $g<19$ mag were observed from the winter of 2008 to the fall of 2009. 
Table 2. Characteristics of the large spectroscopic surveys conducted at the Apache Point Observatory (Apo; New Mexico, USA) in the northern hemisphere with the 2.5-m Sloan Foundation Telescope and at the Las Campanas Observatory (Lco; Atacama, Chile) in the southern hemisphere with the 2.5-m Irénée du Pont Telescope.

\begin{tabular}{|c|c|c|c|}
\hline Name & SEgue-1 & Segue-2 & MARVELS \\
\hline Telescope & \multicolumn{2}{|c|}{ 2.5-m APO } & 2.5-m APO \\
\hline Fibers & \multicolumn{2}{|c|}{ \#640 } & $\# 60$ \\
\hline Wavelengths & \multirow{2}{*}{\multicolumn{2}{|c|}{$\begin{array}{l}385-920 \mathrm{~nm} \\
(H \text {-band })\end{array}$}} & range of $90 \mathrm{~nm}$ \\
\hline & & & (center at $\sim 540 \mathrm{~nm}$ ) \\
\hline Resolution & \multicolumn{2}{|c|}{$\sim 2000$} & \multirow[t]{2}{*}{$\sim 5100$} \\
\hline Survey Area & $1438 \mathrm{deg}^{2}$ & $1317 \mathrm{deg}^{2}$ & \\
\hline \multirow[t]{2}{*}{ Targets } & 240000 stars & 118151 stars & 11 000 main-sequence stars \\
\hline & $(g<19 \mathrm{mag})$ & $(g<19 \mathrm{mag})$ & $\begin{array}{l}\sim 1000 \text { giant stars } \\
(8<V<12 \mathrm{mag})\end{array}$ \\
\hline Date range & 2004-2008 & 2008-2009 & 2008-2014 \\
\hline Name & \multicolumn{2}{|c|}{ APOGEE } & APOGEE-2 \\
\hline Telescope & \multicolumn{2}{|c|}{ 2.5-m APO } & 2.5-m APO \\
\hline Fibers & \multicolumn{2}{|c|}{ \#300 } & \#300 \\
\hline Wavelengths & \multicolumn{2}{|c|}{$\begin{array}{c}1510-1700 \mathrm{~nm} \\
(H \text {-band })\end{array}$} & $\begin{array}{c}1510-1700 \mathrm{~nm} \\
(H \text {-band })\end{array}$ \\
\hline Resolution & \multicolumn{2}{|c|}{$\sim 22500$} & $\sim 22500$ \\
\hline Targets & \multirow{2}{*}{\multicolumn{2}{|c|}{$\begin{array}{c}>100000 \text { giant stars } \\
\quad(H<12.2 \mathrm{mag})\end{array}$}} & \multirow{2}{*}{$\begin{array}{l}>300000 \mathrm{stars} \\
(H<13.5 \mathrm{mag})\end{array}$} \\
\hline & & & \\
\hline Date range & \multicolumn{2}{|c|}{ 2011-2014 } & $2017-2020$ \\
\hline
\end{tabular}

\section{MARVELS}

The Multi-object Apo Radial Velocity Exoplanet Large-area Survey (Marvels; Table 2, top right; http://www.sdss.org/surveys/marvels/; [25]) combined the 2.5-m Sloan Foundation Telescope with two interferometric spectrographs with 60 fibers each. It obtained spectra with a spectral resolution $R \sim 5100$ with a wavelength range of $90 \mathrm{~nm}$ centered at $\sim 540 \mathrm{~nm}$ for about 11000 main sequence stars and about 1000 giant stars with a $V$-magnitude between 8 and 12 mag. For each star, a time-span of 1.5 years was covered with 25 to 35 spectra from which the $v_{\text {rad }}$ could be determined with a typical error of $10 \mathrm{~m} \mathrm{~s}^{-1}$ at $V=10 \mathrm{mag}$. Such observations are ideal to detect gas giant planets with orbital periods ranging from several hours up to two years. The observations were done in bright time from the fall of 2008 until the spring of 2014.

\section{A POGEE $\mathcal{E}$ APOGEE-2}

For the Apache Point Observatory Galactic Evolution Experiment (ApogeE; Table 2, bottom left; http://www.sdss.org/surveys/apogee/; [26]), the 2.5-m Sloan Telescope was combined with a spectrograph with 300 fibers that is capable of obtaining spectra with a resolution of $R \sim 22500$. It observed the region around the $H$-band in the near infrared $(1.51-1.70 \mu \mathrm{m})$. These observations in the northern hemisphere were done in bright time from the spring of 2011 until the spring of 2014. In total, more than 160000 red giant stars across the full range of the Galactic bulge, bar, disk, and halo with a $H$ magnitude below 12.2 were observed. The SNR of the APOGEe spectra is typically above 100 . This is sufficient to derive the stellar parameters $\left(T_{\mathrm{eff}}, \log g,[\mathrm{Fe} / \mathrm{H}]\right)$, the abundances with a pre- 
cision of 0.1 dex for 15 species, and $v_{\text {rad }}$ with an error below $100 \mathrm{~m} \mathrm{~s}^{-1}$. APOGEE also has a sub-project, ApoKasc ([27]), that focuses on stars within the Kepler FoV. Apart from the ApogeE spectroscopic parameters, the ApoKasc catalog also lists asteroseismic values for $\log g$, masses, radii, and mean densities. It is often used as reference work for the quality check of the results of other surveys.

The second phase of the Apache Point Observatory Galaxy Evolution Experiment (APOGEe-2; Table 2, bottom right; http://www.sdss.org/surveys/apogee-2; [28]) aims to study the assembly and formation history of the Milky Way. Spectra with the same characteristics and of similar quality will be obtained for in total more than 300000 stars in the entire Milky Way. Compared to APogeE, the chemical abundance analysis will be extended with 10 species. For planet-hosting stars, the goal is to find out if their chemical composition differs from the one of stars without planets. ApogEe-2 will last for 6 years and only observes during bright time. The observations started in the northern hemisphere in the fall of 2014. After three years, the observations will turn to the south by using the 2.5-m Irénée du Pont Telescope of the Las Campanas Observatory (Atacama, Chile) with a circular FoV with a diameter of $2.1 \mathrm{deg}\left(3.5 \mathrm{deg}^{2}\right)$.

\subsection{Siding Spring Observatory (Australia)}

At the Siding Spring Observatory (Australia) in the southern hemisphere, two telescopes have been used for large spectroscopic surveys (Table 3): the 1.2-m UK Schmidt Telescope (Uks) with a circular FoV with a diameter of $5.7 \mathrm{deg}\left(\sim 25 \mathrm{deg}^{2}\right)$ and the 3.9-m Anglo-Australian Telescope (AAT) with a circular FoV with a diameter of $2 \mathrm{deg}\left(\sim 3 \mathrm{deg}^{2}\right)$.

$R$ AVE

The Radial Velocity Experiment (RAvE; Table 3, left; https://www.rave-survey.org/project; [29]) combined the 1.2-m UK Schmidt Telescope with the multi-object spectrograph $6 \mathrm{dF}$ (6 degree Field) that utilizes interchangeable field plates with 150 fibers each. It enables to observe the wavelength region 841.0-879.5 nm (containing the Ca II triplet) with $R \sim 7500$. The observations were done from April 2003 to July 2005 during 7 nights per lunation, and from August 2005 to April 2013 during 2025 nights per lunation. In total 574630 spectra were gathered for 483330 stars with an I-magnitude between 9 and $13 \mathrm{mag}$. Apart from the basic stellar parameters $\left(T_{\text {eff }}, \log g\right.$, and $\left.[\mathrm{M} / \mathrm{H}]\right)$, also the $v_{\text {rad }}$, distances and elemental abundances $([\mathrm{Fe} / \mathrm{H}]$ and six $\alpha$-elements) were determined with an accuracy of $\sim 1.5 \mathrm{~km} \mathrm{~s}^{-1}, \sim 20 \%$ and $\sim 0.2 \mathrm{dex}$, respectively. Given the large number of observed stars and the big overlap with the Түсно-GAIA asterometric solution catalog ([30]), it was the first spectroscopic survey that allowed to study the Milky Way morphology and history by complementing the RAVE $v_{\text {rad }}$ values with proper motions listed in astrometric databases.

\section{ARGOS}

The Abundances and Radial Velocity Galactic Origins Survey (Argos; Table 3, middle; [31-33]) used the 3.9-m Anglo-Australian Telescope in combination with the AAOmega fibre spectrometer having 400 fibers of which 350 are used for stars. About 28000 red giants with an I-magnitude between 13 and 16 mag and a Galactic latitude near +10 deg or between -10 and -5 deg were observed in two wavelength ranges: a first region including the Ca II triplet $(840-885 \mathrm{~nm})$ with $R \sim 11000$ and a typical $\mathrm{SNR}$ of 75 , and a second region including the $\mathrm{Mgb} / \mathrm{MgH}$ feature $(500-560 \mathrm{~nm}$ ) with a lower spectral resolution of $R \sim 3000$. The latter was included to be able to reject foreground dwarf stars from the sample of more distant giants. In total, 28 fields were covered with 1000 spectra each (except for one with only 600 spectra) during 42 nights in the months May-August of the years 2008-2011. The 
Table 3. Characteristics of the large spectroscopic surveys conducted at the Siding Spring Observatory (Sso; Australia) in the southern hemisphere with the 1.2-m UK Schmidt Telescope (Uкs) and the 3.9-m Anglo-Australian Telescope (ААт).

\begin{tabular}{|c|c|c|c|}
\hline Name & RAVE & ARGOS & GalaH \\
\hline $\begin{array}{l}\text { Telescope } \\
\text { Fibers }\end{array}$ & $\begin{array}{c}\text { 1.2-m Uks@Sso } \\
\# 150\end{array}$ & $\begin{array}{c}\text { 3.9-m AAT@Sso } \\
\text { \#400 }\end{array}$ & $\begin{array}{c}\text { 3.9-m AAT@Sso } \\
\text { \#400 }\end{array}$ \\
\hline Wavelengths & $\begin{array}{l}841.0-879.5 \mathrm{~nm} \\
\text { (Ca II triplet) }\end{array}$ & $\begin{array}{cc}840-885 \mathrm{~nm} & 500-560 \mathrm{~nm} \\
(\mathrm{Ca} \text { II triplet }) & (\mathrm{Mgb} / \mathrm{MgH})\end{array}$ & $\begin{array}{l}471.8-490.3 \mathrm{~nm} \\
564.9-587.3 \mathrm{~nm} \\
648.1-673.9 \mathrm{~nm} \\
759.0-789.0 \mathrm{~nm}\end{array}$ \\
\hline Resolution & $\sim 7500$ & $\sim 11000$ & $\sim 28000$ \\
\hline $\begin{array}{l}\text { Targets } \\
\text { Date range }\end{array}$ & $\begin{array}{c}483330(\text { stars }) \\
\left(\begin{array}{c}9<I<13 \mathrm{mag}) \\
2003-2013\end{array}\right.\end{array}$ & $\begin{array}{c}\sim 28000 \text { red giants } \\
(13<I<16 \mathrm{mag}) \\
2008-2011\end{array}$ & $\begin{array}{c}1000000 \text { stars } \\
(12<V<14 \mathrm{mag}) \\
2012-2017\end{array}$ \\
\hline
\end{tabular}

main reason to study these stars in the Galactic bulge and inner disk was to find out more about the formation processes of the bulge.

\section{GALAH}

The Galactic Archaeology with Hermes Survey (GalaH; Table 3, right; https://galah-survey .org/; [34-37]) combines the 3.9-m Anglo-Australian Telescope with the High Efficiency and Resolution Multi-Element Spectrograph (Hermes). It has 400 fibers and observes in four wavelength regions: 471.8-490.3 nm (blue), 564.9-587.3 nm (green), 648.1-673.9 nm (red), and 759.0-789.0 nm (IR). The spectra with a resolution of $R \sim 28000$ and a required SNR of 100 are being used to derive the chemical abundances of 30 elements with an accuracy of 0.05 dex. In total, about 1000000 stars with a $V$-magnitude between 12 and $14 \mathrm{mag}$ and a declination below $10 \mathrm{deg}$ are being observed. They originate from different parts of our galaxy: $77 \%$ thin-disk stars, $22 \%$ thick-disk stars, $0.8 \%$ bulge stars, and $0.2 \%$ halo stars. The observations started in 2012 and will end in 2017.

\subsection{GAIA related}

\section{GAIA}

The Global Astrometric Interferometer for Astrophysics (GAIA; Table 4, left; (http://www.esa.int/ Our_Activities/Space_Science/Gaia; [6]) is a space mission with a $1.45 \times 0.5 \mathrm{~m}^{2}$ size telescope onboard. It performs photometric, astrometric and spectroscopic observations of objects passing through its FoV of $0.22 \times 0.39 \mathrm{deg}^{2}$. GaIA was launched on December 19, 2013 and started its scientific observations in sky scanning mode on August 22, 2014. During the 5.5 years of the nominal mission, it is expected to gather data for approximately 150000000 objects (but rumour has it that the mission will be extended). For the spectroscopic part, the Radial Velocity Spectrometer (Rvs) is used to obtain spectra with $R \sim 11500$ in the wavelength range surrounding the Ca II triplet (847-874 nm). The aimed precision of the $v_{\text {rad }}$ determinations ranges from $1 \mathrm{~km} \mathrm{~s}^{-1}$ for stars with $V=11.5$ mag to $30 \mathrm{~km} \mathrm{~s}^{-1}$ for stars with $V=17.5 \mathrm{mag}$. For stars with a $V$-magnitude up to $15 \mathrm{mag}$, also the atmospheric parameters will be provided in the output catalogue. 
Table 4. Characteristics of the large spectroscopic surveys related to the GaIA space-mission conducted in space with the Radial Velocity Spectrometer (Rvs) and at the European Southern Observatory (Eso; Paranal, Chile) with the 8.2-m Very Large Telescope (VLT/UT2) in the southern hemisphere.

\begin{tabular}{|l|c|cc|}
\hline Name & GAIA & \multicolumn{2}{|c|}{ GAIA-Eso } \\
\hline Telescope & $1.45 \mathrm{~m} \times 0.5 \mathrm{~m}$ & \multicolumn{2}{|c|}{8.2 -m VLT $@$ Eso } \\
Instrument & Rvs & GIRAFFE & UvES \\
Fibers & & $\# 132$ & $\# 8$ \\
Wavelengths & $847-874 \mathrm{~nm}$ & $370-950 \mathrm{~nm}$ & $300-1100 \mathrm{~nm}$ \\
& $($ Ca II triplet $)$ & \multicolumn{2}{|c}{} \\
Resolution & $\sim 11500$ & $7500-30000 \quad \sim 40000$ \\
Targets & $\sim 150000000 \mathrm{stars}$ & \multicolumn{2}{|c}{$>10000$ stars } \\
& $(V<17.5 \mathrm{mag})$ & $(V<19 \mathrm{mag}) \quad(V<16.5 \mathrm{mag})$ \\
Date range & $2014-2020$ & \multicolumn{2}{|c}{$2012-2016$} \\
\hline
\end{tabular}

\section{GAIA-ESO}

The GaIA-Eso survey (Table 4, right; https://www.gaia-eso.eu/; [38]) is a ground-based spectroscopic support effort for the GAIA mission. It is a large observing program based in the southern hemisphere at the European Southern Observatory (Eso) that runs on the second unit (UT2) of the 8.2-m Very Large Telescope (VLT) in Paranal (Chile). This telescope only has a small, circular FoV with a diameter of $25 \mathrm{arcmin}\left(\sim 0.135 \mathrm{deg}^{2}\right)$. The observations are done with the Fibre Large Array Multi Element Spectrograph (FLAMES) feeding two spectrographs covering the whole visual spectral range. GIRAFFE is equipped with 130 fibers and is used to obtain spectra with a spectral resolution in the range of 7500 to 30000 for stars with $V<19$ mag. Spectra with $R \sim 40000$ are collected for up to 8 stars with $V<16.5$ mag simultaneously with the Ultraviolet and Visual Echelle Spectrograph (Uves). The observations of the Gaia-Eso survey started on December 31, 2011 and ended recently after a period of 5 years. In total, more than 10000 Milky Way stars, both in the field and in open clusters, were observed. Their astrophysical parameters, including detailed abundances for at least 12 elements ( $\mathrm{Na}, \mathrm{Mg}, \mathrm{Si}, \mathrm{Ca}, \mathrm{Ti}, \mathrm{V}, \mathrm{Cr}, \mathrm{Mn}, \mathrm{Fe}, \mathrm{Co}, \mathrm{Sr}, \mathrm{Zr}, \mathrm{Ba})$ and $v_{\text {rad }}$ values with a precision between 0.1 and $5 \mathrm{~km} \mathrm{~s}^{-1}$, are being derived as we speak.

\section{Future large spectroscopic surveys}

In the next few years, there are some large spectroscopic surveys planned. They all serve as groundbased spectroscopic support for current and/or future space missions. In this section, three of them are briefly introduced.

\section{WEAVE}

The WHT Enhanced Area Velocity Explorer (WeAvE; http://www.ing.iac.es/weave/about.html; [39]) is designed to use an upgraded version of the 4.2-m William Herschell Telescope (WHT), located in the Observatorio Roque de los Muchachos (Orm; La Palma, Canary Islands, Spain) in the northern hemisphere. The new optics that will be installed will increase its circular FoV to a diameter of $2 \mathrm{deg}\left(\sim 3 \mathrm{deg}^{2}\right)$. A total 1000 fibers will be installed to obtain spectra with $R \sim 5000$ in the full optical range (370-1000 nm) or with $R \sim 20000$ with a limited wavelength coverage. WeAVE aims to deliver more accurate $v_{\text {rad }}$ values than those that will be delivered by GaIA for some 1000000 stars to investigate how the Milky Was was assembled. It will also observe distant galaxies to deduce their 
Table 5. Characteristics of the large spectroscopic surveys that will be conducted at the Observatorio Roque de los Muchachos (Orm; La Palma, Canary Islands, Spain) in the northern hemisphere with the 4.2-m William Herschel Telescope (Wнт) and at the European Southern Observatory (Eso; Paranal, Chile) in the southern hemisphere with the 8.2-m Very Large Telescope (VLT) and the 4.1-m VISTA telescope.

\begin{tabular}{|c|c|c|c|}
\hline Name & Weave & MoOns & 4Most \\
\hline Telescope & 4.2-m WHT@ORM & 8.2-m VtT@Eso & 4.1-m VISTA@Eso \\
\hline Hemisphere & north & south & south \\
\hline FoV & $\sim 3 \operatorname{deg}^{2}$ & $\sim 0.135 \mathrm{deg}^{2}$ & $\sim 4.1 \mathrm{deg}^{2}$ \\
\hline & (2 deg; circular) & (25 arcmin; circular) & (hexagonal) \\
\hline Fibers & $\# 1000$ & \#1024 & \#1624 \\
\hline Wavelengths & $370-1000 \mathrm{~nm}$ & $640-1800 \mathrm{~nm}$ & $392.6-435.5 \mathrm{~nm}$ \\
\hline & & & $\begin{array}{l}516.0-573.0 \mathrm{~nm} \\
610.0-679.0 \mathrm{~nm}\end{array}$ \\
\hline Resolution & $\sim 5000$ & 4 000-6 000 (full) & $<5000$ \\
\hline & $\sim 20000$ & 〜9000 (770-900 nm) & (HRM) \\
\hline & & $\sim 20000(1520-1630 \mathrm{~nm})$ & \\
\hline Targets & $\sim 1000000$ stars & & $\begin{array}{c}\text { stars with }-70<\text { DEC }<+30 \text { deg } \\
(>25000000 \text { spectra })\end{array}$ \\
\hline Date range & from 2018 onwards & from 2019 onwards & $\begin{array}{l}\text { from 2020-2022 onwards } \\
\text { (5 years) }\end{array}$ \\
\hline
\end{tabular}

chemical composition and the rate at which new stars were forming at the time we are seeing them. By determining $v_{\text {rad }}$ values for serveral million galaxies, WEAVE will make it possible to look for small deviations from uniformity in the expansion of the Universe, and hence to get some clues about dark energy. First light of Weave is expected in March 2018 and the start of its science observations is currently scheduled for June 2018.

\section{MoONS}

The Multi-Object Optical and Near-infrared Spectrograph (Moons; http://www.roe.ac.uk/ ciras/ MOONS/VLT-MOONS.html; [40]) is a third generation instrument for the 8.2-m VLT in Paranal (Chile) in the southern hemisphere. With its full wavelength range being $640-1800 \mathrm{~nm}$, it will extend the observations to the short-wavelength infrared. Moons will be offered in two resolution modes. In one mode, spectra with $R$ ranging from $\sim 4000$ to $\sim 6000$ will be obtained in the full wavelength range. In the other mode, spectra with a higher $R$ will be gathered in small wavelength regions: the region of the Ca II triplet (770-900 nm) with $R \sim 9000$ (to measure $v_{\text {rad }}$ ) and part of the $H$-band in the infrared (1520-1630 nm) with $R \sim 20000$ (for a detailed abundance analysis). It will be a powerful tool to perform pioneering research in a wide range of galactic, extragalactic and cosmological studies. Moreover, it will provide crucial follow-up observations for space missions like GAIA ([6]) and Euclid ([41]), and ground-based facilities like Lsst ([42]). Moons is expected to perform scientific observations from 2019 onwards.

\section{$4 M$ ost}

The 4-metre Multi-Object Spectroscopic Telescope (4Most; https://www.4most. eu/; [43]) will use the 4.1-m Vista telescope of Eso in Paranal (Chile) in the southern hemisphere. The hexagonal FoV of $4.1 \mathrm{deg}^{2}$ will be covered with 2436 fibers. It will use three spectrographs with 812 fibers each: 


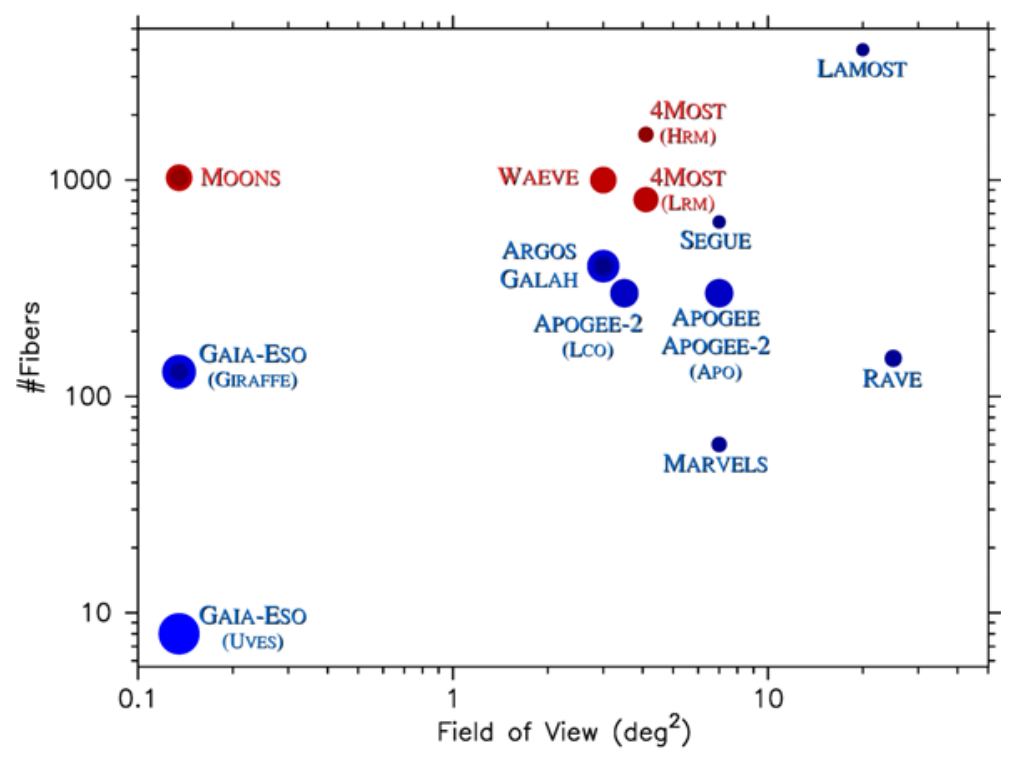

Figure 1. The number of fibers relative to the size of the field of view for the instrument/telescope combinations as used for the large spectroscopic surveys that are discussed in this review. The past/ongoing and future large spectroscopic surveys are given with dots in shades of blue and red, respectively. The size of the dots and their colour brightness are proportional to the (maximum) spectral resolution of the surveys. For the meaning of the abbreviations, we refer to the text and to Tables 1-5.

two to gather spectra in low-resolution mode (LRM) with $R<5000$ in the full optical range (370$950 \mathrm{~nm}$ ) and one to obtain spectra in high-resolution mode (НRM) with a $R>18000$ in three small wavelength ranges $(392.6-435.5 \mathrm{~nm}, 516.0-573.0 \mathrm{~nm}$, and $610.0-679.0 \mathrm{~nm})$. The main aim of 4MosT is to complement the all-sky space missions GAIA ([6]), EucLID ([41]), and eRosita ([44]) by providing $v_{\text {rad }}$ values with an accuracy below $2 \mathrm{~km} \mathrm{~s}^{-1}$ for faint stars observed by GAIA $(r<19$ mag), abundances of up to 15 chemical elements for stars with $V \leq 16 \mathrm{mag}$, and redshifts of galaxies and active galactic nuclei with $r \leq 22 \mathrm{mag}$. In total, more than 25000000 spectra will be collected in a timespan of 5 years for targets spread over a large fraction of the southern sky (declination between -70 and $+30 \mathrm{deg}$ ). 4Most will obtain $R>18000$ resolution spectra for nearly all stars with $V<12$ mag in that area every $\sim 2$ years. The observations are expected to start somewhere in 2020-2022.

\section{Summary and conclusions}

Large spectroscopic surveys are extremely useful for the research of pulsating stars. In general, they make the largest contribution by providing accurate values of stellar parameters and radial velocities. In this review, we have introduced 11 past or ongoing surveys (Lamost, ApogeE, Apogee-2, Marvels, Segue-1, Segue-2, Rave, Argos, Galah, Gaia, and Gaia-Eso) and 3 future surveys (Weave, Moons, and 4Most) that are based in 3 northern observatories (XING, Apo, ORM), 3 southern observatories (Lco, Sso, Eso) and one in space (GAIA). All those that are based on Earth use multi-fiber spectrographs to increase the efficiency of the data gathering process. In Figure 1, we show how the number of fibers relates to the FoV of the telescopes. Lamost is the instrument with the highest number of fibers while RAvE uses the telescope with the largest FoV. Moons, that will use the 8.2-m VLT@Eso, 


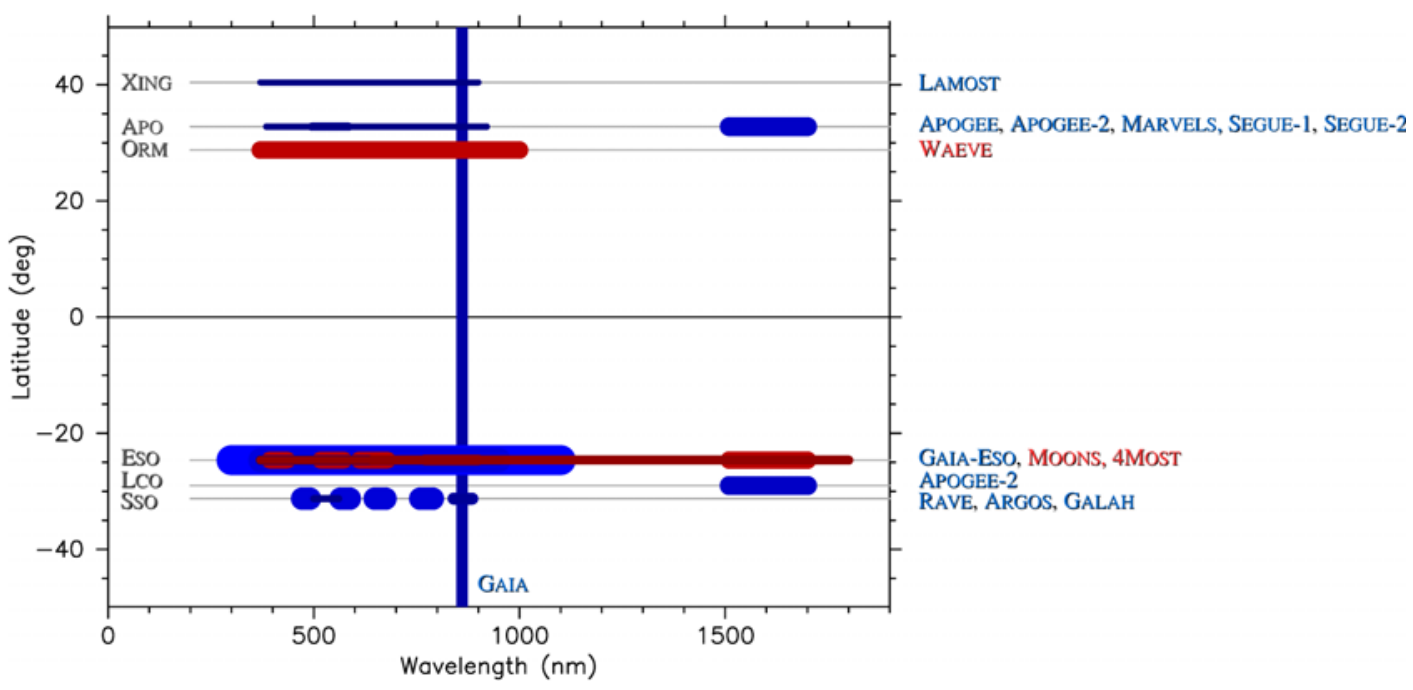

Figure 2. Overview of the spectral ranges covered from the different observatories by the large spectroscopic surveys that are discussed in this review. The space-based mission GaIA is given by the vertical line. The past/ongoing and future large spectroscopic surveys are given with lines in shades of blue and red, respectively. The width of the lines and the brightness of their colour are proportional to the (maximum) spectral resolution of the surveys. For the meaning of the abbreviations, we refer to the text and to Tables 1-5.

will have by far the highest number of fibers per $\operatorname{deg}^{2}$ on the sky. Figure 2 shows that the large spectroscopic surveys discussed in this review have wavelength ranges starting in the near ultraviolet $(300 \mathrm{~nm})$ and ending in the short-wavelength infrared $(1.8 \mu \mathrm{m})$. When combining all of them, those based in the southern hemisphere cover the full wavelength region. In the northern hemisphere, there is no survey that included/includes/will include the range from $1 \mu \mathrm{m}$ to $1.51 \mu \mathrm{m}$.

A huge amount of spectroscopic data of large spectroscopic surveys is publicly available and this amount is steadily increasing. Therefore, it might be worthwhile to check if the spectroscopic data that you might need for the research of your favorite star(s) is already available in the spectroscopic databases before applying for precious telescope time.

Acknowledgments: The author is very thankful to the organisers for the invitation to present this review.

\section{References}

[1] G. Walker, J. Matthews, R. Kuschnig, R. Johnson, S. Rucinski, J. Pazder, G. Burley, A. Walker, K. Skaret, R. Zee et al., PASP 115, 1023 (2003)

[2] A. Baglin, M. Auvergne, L. Boisnard, T. Lam-Trong, P. Barge, C. Catala, M. Deleuil, E. Michel, W. Weiss, CoRoT: a high precision photometer for stellar ecolution and exoplanet finding, in 36th COSPAR Scientific Assembly (2006), Vol. 36 of COSPAR Meeting

[3] W.J. Borucki, D. Koch, G. Basri, N. Batalha, T. Brown, D. Caldwell, J. Caldwell, J. ChristensenDalsgaard, W.D. Cochran, E. DeVore et al., Science 327, 977 (2010)

[4] S.B. Howell, C. Sobeck, M. Haas, M. Still, T. Barclay, F. Mullally, J. Troeltzsch, S. Aigrain, S.T. Bryson, D. Caldwell et al., PASP 126, 398 (2014) 
[5] W.W. Weiss, S.M. Rucinski, A.F.J. Moffat, A. Schwarzenberg-Czerny, O.F. Koudelka, C.C. Grant, R.E. Zee, R. Kuschnig, S. Mochnacki, J.M. Matthews et al., PASP 126, 573 (2014)

[6] M.A.C. Perryman, K.S. de Boer, G. Gilmore, E. Høg, M.G. Lattanzi, L. Lindegren, X. Luri, F. Mignard, O. Pace, P.T. de Zeeuw, A\&A 369, 339 (2001)

[7] G.R. Ricker, J.N. Winn, R. Vanderspek, D.W. Latham, G.Á. Bakos, J.L. Bean, Z.K. BertaThompson, T.M. Brown, L. Buchhave, N.R. Butler et al., Journal of Astronomical Telescopes, Instruments, and Systems 1, 014003 (2015)

[8] H. Rauer, C. Catala, C. Aerts, T. Appourchaux, W. Benz, A. Brandeker, J. ChristensenDalsgaard, M. Deleuil, L. Gizon, M.J. Goupil et al., Experimental Astronomy 38, 249 (2014)

[9] T.R. Bedding, H. Kjeldsen, Communications in Asteroseismology 161, 3 (2010)

[10] T.R. Bedding, S.J. Murphy, I.L. Colman, D.W. Kurtz, Échelle diagrams and period spacings of g modes in $\gamma$ Doradus stars from four years of Kepler observations, in European Physical Journal Web of Conferences (2015), Vol. 101 of European Physical Journal Web of Conferences, p. 01005

[11] M.A. Dupret, J. De Ridder, P. De Cat, C. Aerts, R. Scuflaire, A. Noels, A. Thoul, A\&A 398, 677 (2003)

[12] M. Briquet, C. Aerts, A\&A 398, 687 (2003)

[13] W. Zima, A\&A 455, 227 (2006)

[14] P. Pietrukowicz, M. Latour, R. Angeloni, F. di Mille, I. Soszyński, A. Udalski, C. Germanà, AcA 65, 63 (2015)

[15] P.I. Pápics, E. Moravveji, C. Aerts, A. Tkachenko, S.A. Triana, S. Bloemen, J. Southworth, A\&A 570, A8 (2014)

[16] X.Q. Cui, Y.H. Zhao, Y.Q. Chu, G.P. Li, Q. Li, L.P. Zhang, H.J. Su, Z.Q. Yao, Y.N. Wang, X.Z. Xing et al., Research in Astronomy and Astrophysics 12, 1197 (2012)

[17] A.L. Luo, Y.H. Zhao, G. Zhao, L.C. Deng, X.W. Liu, Y.P. Jing, G. Wang, H.T. Zhang, J.R. Shi, X.Q. Cui et al., Research in Astronomy and Astrophysics 15, 1095 (2015)

[18] L.C. Deng, H.J. Newberg, C. Liu, J.L. Carlin, T.C. Beers, L. Chen, Y.Q. Chen, N. Christlieb, C.J. Grillmair, P. Guhathakurta et al., Research in Astronomy and Astrophysics 12, 735 (2012)

[19] P. De Cat, J.N. Fu, A.B. Ren, X.H. Yang, J.R. Shi, A.L. Luo, M. Yang, J.L. Wang, H.T. Zhang, H.M. Shi et al., ApJS 220, 19 (2015)

[20] A. Ren, J. Fu, P. De Cat, Y. Wu, X. Yang, J. Shi, A. Luo, H. Zhang, S. Dong, R. Zhang et al., ApJS 225, 28 (2016)

[21] A. Frasca, J. Molenda-Żakowicz, P. De Cat, G. Catanzaro, J.N. Fu, A.B. Ren, A.L. Luo, J.R. Shi, Y. Wu, H.T. Zhang, A\&A 594, A39 (2016)

[22] R.O. Gray, C.J. Corbally, P. De Cat, J.N. Fu, A.B. Ren, J.R. Shi, A.L. Luo, H.T. Zhang, Y. Wu, Z. Cao et al., AJ 151, 13 (2016)

[23] J. Fu, P. De Cat, M. Smith, Synergies between spectroscopic and time-series photometric surveys - LAMOST observations for the Kepler field and K2 fields, in American Astronomical Society Meeting Abstracts (2017), Vol. 229 of American Astronomical Society Meeting Abstracts, p. 305.02

[24] B. Yanny, C. Rockosi, H.J. Newberg, G.R. Knapp, J.K. Adelman-McCarthy, B. Alcorn, S. Allam, C. Allende Prieto, D. An, K.S.J. Anderson et al., AJ 137, 4377-4399 (2009)

[25] J. Ge, S. Mahadevan, B. Lee, X. Wan, B. Zhao, J. van Eyken, S. Kane, P. Guo, E. Ford, S. Fleming et al., The Multi-object APO Radial-Velocity Exoplanet Large-area Survey (MARVELS), in Extreme Solar Systems, edited by D. Fischer, F.A. Rasio, S.E. Thorsett, A. Wolszczan (2008), Vol. 398 of Astronomical Society of the Pacific Conference Series, p. 449 
[26] C. Allende Prieto, S.R. Majewski, R. Schiavon, K. Cunha, P. Frinchaboy, J. Holtzman, K. Johnston, M. Shetrone, M. Skrutskie, V. Smith et al., Astronomische Nachrichten 329, 1018 (2008)

[27] M.H. Pinsonneault, Y. Elsworth, C. Epstein, S. Hekker, S. Mészáros, W.J. Chaplin, J.A. Johnson, R.A. García, J. Holtzman, S. Mathur et al., ApJS 215, 19 (2014)

[28] S.R. Majewski, APOGEE Team, APOGEE-2 Team, Astronomische Nachrichten 337, 863 (2016)

[29] M. Steinmetz, T. Zwitter, A. Siebert, F.G. Watson, K.C. Freeman, U. Munari, R. Campbell, M. Williams, G.M. Seabroke, R.F.G. Wyse et al., AJ 132, 1645 (2006)

[30] ESA, ed., The HIPPARCOS and TYCHO catalogues. Astrometric and photometric star catalogues derived from the ESA HIPPARCOS Space Astrometry Mission, Vol. 1200 of ESA Special Publication (1997)

[31] K. Freeman, M. Ness, E. Wylie-de-Boer, E. Athanassoula, J. Bland-Hawthorn, M. Asplund, G. Lewis, D. Yong, R. Lane, L. Kiss et al., MNRAS 428, 3660 (2013)

[32] M. Ness, K. Freeman, E. Athanassoula, E. Wylie-de-Boer, J. Bland-Hawthorn, M. Asplund, G.F. Lewis, D. Yong, R.R. Lane, L.L. Kiss, MNRAS 430, 836 (2013)

[33] M. Ness, K. Freeman, E. Athanassoula, E. Wylie-de-Boer, J. Bland-Hawthorn, M. Asplund, G.F. Lewis, D. Yong, R.R. Lane, L.L. Kiss et al., MNRAS 432, 2092 (2013)

[34] G.M. De Silva, K.C. Freeman, J. Bland-Hawthorn, S. Martell, E.W. de Boer, M. Asplund, S. Keller, S. Sharma, D.B. Zucker, T. Zwitter et al., MNRAS 449, 2604 (2015)

[35] J.D. Simpson, G.M. De Silva, J. Bland-Hawthorn, K.C. Freeman, S.L. Martell, K.J. Schlesinger, S. Sharma, D.B. Zucker, T. Zwitter, J. Kos et al., MNRAS 459, 1069 (2016)

[36] J. Kos, J. Lin, T. Zwitter, M. Žerjal, S. Sharma, J. Bland-Hawthorn, M. Asplund, A.R. Casey, G.M. De Silva, K.C. Freeman et al., MNRAS 464, 1259 (2017)

[37] S.L. Martell, S. Sharma, S. Buder, L. Duong, K.J. Schlesinger, J. Simpson, K. Lind, M. Ness, J.P. Marshall, M. Asplund et al., MNRAS 465, 3203 (2017)

[38] G. Gilmore, S. Randich, M. Asplund, J. Binney, P. Bonifacio, J. Drew, S. Feltzing, A. Ferguson, R. Jeffries, G. Micela et al., The Messenger 147, 25 (2012)

[39] G. Dalton, S.C. Trager, D.C. Abrams, D. Carter, P. Bonifacio, J.A.L. Aguerri, M. MacIntosh, C. Evans, I. Lewis, R. Navarro et al., WEAVE: the next generation wide-field spectroscopy facility for the William Herschel Telescope, in Ground-based and Airborne Instrumentation for Astronomy IV (2012), Vol. 8446 of Proc. SPIE, p. 84460P

[40] M. Cirasuolo, MOONS Consortium, MOONS: A New Powerful Multi-Object Spectrograph for the VLT, in Multi-Object Spectroscopy in the Next Decade: Big Questions, Large Surveys, and Wide Fields, edited by I. Skillen, M. Barcells, S. Trager (2016), Vol. 507 of Astronomical Society of the Pacific Conference Series, p. 109

[41] L. Amendola, S. Appleby, D. Bacon, T. Baker, M. Baldi, N. Bartolo, A. Blanchard, C. Bonvin, S. Borgani, E. Branchini et al., Living Reviews in Relativity 16, 6 (2013)

[42] LSST Science Collaboration, P.A. Abell, J. Allison, S.F. Anderson, J.R. Andrew, J.R.P. Angel, L. Armus, D. A(rnett, S.J. Asztalos, T.S. Axelrod et al., ArXiv e-prints (2009), 0912.0201

[43] R.S. de Jong, 4MOST Consortium, 4MOST Spectroscopy Consortium, Astronomische Nachrichten 337, 964 (2016)

[44] A. Merloni, P. Predehl, W. Becker, H. Böhringer, T. Boller, H. Brunner, M. Brusa, K. Dennerl, M. Freyberg, P. Friedrich et al., ArXiv e-prints (2012), 1209. 3114 